\title{
TAMANHO DE RIZOMAS-SEMENTE E FILEIRAS DE PLANTAS NO CANTEIRO NA PRODUÇÃO DO MANGARITO CV. COMUM
}

\author{
Size of seed-rhizomes and rows of plants on Comum tannia yield
}

\author{
Néstor Antônio Heredia Zárate ${ }^{1}$, Maria do Carmo Vieira², \\ Marcelo Helmich ${ }^{3}$, Márcio Antonio Maria ${ }^{3}$
}

\begin{abstract}
RESUMO
O objetivo do trabalho foi avaliar a capacidade produtiva do mangarito, cv. Comum (Xanthosoma mafaffa Schott), proveniente de rizomas-semente secundários de tamanhos diferentes, cultivados sob três ou quatro fileiras de plantas no canteiro. Os tratamentos foram constituídos pela combinação do número de fileiras de plantas no canteiro e do plantio de quatro tamanhos de rizomassemente secundários (massa fresca, em grama, e diâmetro, em milímetro, respectivamente, de $\mathrm{T}_{1}=2,96$ e $8,77, \mathrm{~T}_{2}=1,33$ e $6,05, \mathrm{~T}_{3}=$ 1,13 e 5,68 e $\mathrm{T}_{4}=0,78$ e 4,83, médias de 300 rizomas de cada tamanho), como fatorial $2 \times 4$, no delineamento experimental de blocos casualizados, com quatro repetições. A colheita foi realizada aos 300 dias após o plantio. As produções de massa fresca dos rizomas, total, rizomas-mãe e rizomas-filho, foram influenciadas significativamente pela interação número de fileiras de plantas no canteiro e tamanho dos rizomas-semente. As médias dos diâmetros, comprimentos e massas frescas dos rizomas-mãe (43,13 mm; $21,36 \mathrm{~mm}$ e $13,21 \mathrm{~g})$ e dos rizomas-filho, nas classes grande $(37,15 \mathrm{~mm} ; 18,57 \mathrm{~mm}$ e $9,60 \mathrm{~g})$, média $(30,71 \mathrm{~mm} ; 14,18 \mathrm{~mm}$ e 4,14 g) e pequena $(19,72 \mathrm{~mm} ; 9,46 \mathrm{~mm}$ e 1,07 g), apresentaram variações significativas dependentes da interação entre número de fileiras no canteiro e tamanho de rizomas-semente, dentro de cada característica avaliada nos rizomas. As maiores produções totais (11,48 $\left.\mathrm{t} \mathrm{ha}^{-1}\right)$ e de rizomas comerciais $\left(4,51 \mathrm{t} \mathrm{ha}^{-1}\right)$ foram do tratamento três fileiras de plantas e rizomas-semente $\mathrm{T}_{1}$ e as de rizomas nãocomerciais foram do tratamento quatro fileiras de plantas e rizomas-semente $T_{2}\left(7,41 \mathrm{t} \mathrm{ha}^{-1}\right)$. As menores produções totais $(0,96$ $\left.\mathrm{t} \mathrm{ha}^{-1}\right)$, líquida $\left(0,86 \mathrm{t} \mathrm{ha}^{-1}\right)$ e de rizomas comerciais $\left(0,60 \mathrm{t} \mathrm{ha}^{-1}\right)$ e não-comerciais $\left(0,26 \mathrm{t} \mathrm{ha}^{-1}\right)$ foram do tratamento três fileiras de plantas e plantio de rizomas $\mathrm{T}_{4}$.
\end{abstract}

Termos para indexação: Xanthosoma mafaffa, propágulos, produtividade.

\begin{abstract}
The objective of this work was to evaluate yield capacity of Comum tannia (Xanthosoma mafaffa Schott) from secondary seed-rhizomes of different sizes and cultivated under three or four rows of plants per plot. Treatments were established by the combination of the number of rows of plants per plot and by the planting of four sizes of secondary seed-rhizomes (fresh mass, in gram, and diameter, in millimeter, respectively, of $\mathrm{T}_{1}=2.96$ and 8.77; $\mathrm{T}_{2}=1.33$ and $6.05 ; \mathrm{T}_{3}=1.13$ and 5.68 and $\mathrm{T}_{4}=0.78$ and 4.83 , average of 300 rhizomes of each size), as 2 x 4 factorial scheme in a randomized block experimental design with four replications. Harvest was done on 300 days after planting. Yields of fresh mass of rhizomes, total, corms and cormels were influenced significantly by number of rows per plot/size of seed-rhizomes interaction. Average of diameters, lengths and fresh masses of corms (43.13 mm; $21.36 \mathrm{~mm}$ and $13.21 \mathrm{~g})$ and cormels, from big $(37.15 \mathrm{~mm} ; 18.57 \mathrm{~mm}$ and $9.60 \mathrm{~g})$, medium $(30.71 \mathrm{~mm} ; 14.18 \mathrm{~mm}$ and $4.14 \mathrm{~g})$ and small $(19.72 \mathrm{~mm} ; 9.46 \mathrm{~mm}$ and $1.07 \mathrm{~g})$ classes, showed variations significantly which were dependent on number of rows per plot/size of seed-rhizome interaction, inside each evaluated characteristic of rhizomes. The highest total yields $\left(11.48 \mathrm{t} \mathrm{ha}^{-1}\right)$ and commercial rhizomes $\left(4.51 \mathrm{t} \mathrm{ha}^{-1}\right)$ were from the treatment with three rows of plants and $\mathrm{T}_{1}$ seed-rhizome and the highest yields of noncommercial rhizomes were from the treatment with four rows of plants and $\mathrm{T}_{2}$ seed-rhizome $\left(7.41 \mathrm{t} \mathrm{ha}^{-1}\right)$. The most low total yields $\left(0.96 \mathrm{t} \mathrm{ha}^{-1}\right)$, net $\left(0.86 \mathrm{t} \mathrm{ha}^{-1}\right)$, commercial $\left(0.60 \mathrm{tha}^{-1}\right)$ and of non-commercial $\left(0.26 \mathrm{t} \mathrm{ha}^{-1}\right)$ were from the treatment with three rows of plants and the planting of $\mathrm{T}_{4}$ rhizomes.
\end{abstract}

Index terms: Xanthosoma mafaffa, propagules, productivity.

\section{(Recebido para publicação em 3 de março de 2005 e aprovado em 5 de abril de 2006)}

\section{INTRODUÇÃO}

Durante o curso da evolução, as plantas se adaptaram a extremos de ambientes terrestres, desenvolvendo ampla diversidade de formas de vida altamente especializadas, freqüentemente, envolvendo modificações drásticas da estrutura e o funcionamento de órgãos e tecidos específicos do corpo vegetal. Sistemas subterrâneos espessados, como tubérculos, bulbos, rizomas e outros que acumulam compostos de reserva constituem verdadeiros depósitos energéticos, que são utilizados tanto pelo homem, como fonte de carbono, energia e matérias-primas, como pelas próprias plantas, para o desenvolvimento das gemas durante a brotação (APPEZZATO-DA-GLÓRIA, 1996).

${ }^{1}$ Professor do departamento de Ciências Agrárias/DCA - Universidade Federal do Mato Grosso do Sul/UFMS - Cx.P. 533 - $79804-970$ - Dourados, MS Bolsista de Produtividade em Pesquisa do CNPq - nheredia@ceud.ufms.br

2Professora do Departamento de Ciências Agrárias/DCA - Cx.P. 533 - 79804-970 - Dourados, MS - Bolsista de Produtividade em Pesquisa do CNPq. ${ }^{3}$ Bolsista de Iniciação Científica FUNDECT/CNPq. 
As culturas tuberosas incluem um grande número de espécies de plantas rústicas, que são muito disseminadas nas regiões tropicais do globo terrestre. A grande maioria dessas espécies é de amiláceas, isto é, produtoras de materiais nos quais predomina o amido como componente. São classificadas como calóricas e consideradas como alimentos de subsistência, capazes de proporcionar energia para populações carentes. Entre as espécies mais energéticas estão a mandioca (Manihot esculenta Crantz), a mandioquinha-salsa (Arracacia xanthorrhiza Bancroft) e o mangarito (Xanthosoma mafaffa Schott), com 142,0, 125,0 e 107,2 kcal/100 g, respectivamente (CEREDA, 2002).

Pelas características culinárias peculiares de seus rizomas, o mangará, também conhecido por mangarito, é muito apreciado pela população rural de São Paulo e só é comercializado, sazonalmente, nos locais próximos às áreas de produção. Todavia, progressos tecnológicos que levem ao aumento da produção e da qualidade comercial dos rizomas, poderão torná-lo um produto mais popular para o mercado hortigranjeiro nacional.

Um dos fatores que tem limitado a expansão de várias espécies propagadas vegetativamente, segundo Sediyama \& Casali (1997), é a falta de material de plantio e por isso é recomendado o bom aproveitamento das mudas. Para essas espécies, o tipo e a qualidade do material de plantio determinam as diferenças na velocidade de enraizamento, no crescimento e, conseqüentemente, na produção e extensão do ciclo vegetativo. Vários aspectos das mudas ainda não foram estudados e, se o foram, os resultados ainda não são conclusivos. Vasconcelos (1972), citado por Monteiro \& Peressin (1997), estabeleceu-se que os rizomas-semente do mangarito, do tipo primário (rizomas-mãe), com massa fresca variando de $13 \mathrm{~g}$ a $40 \mathrm{~g}$, foram mais produtivos que os rizomassemente secundários (rizomas-filho), com 1,5 a 4,5 g. Os rizomas primários obtidos na cultura anterior, além dos problemas fitossanitários, dificilmente são o bastante, em termos numéricos, para a implantação da nova cultura.

Monteiro \& Peressin (1997) estudaram o efeito de dois tamanhos de rizomas-semente secundários (grande = $5 \mathrm{~g}$ e pequeno $=2 \mathrm{~g}$ ), em três épocas de plantio (início, meados e fim de outubro de 1985) e em três locais do Estado de São Paulo (Serra Negra, Monte Alegre do Sul e Itu), sem a utilização de irrigação complementar. Em geral, os rizomassemente do tipo grande superaram os pequenos em termos de produção e houve interações entre o tamanho dos rizomas-semente e a época de plantio. Também observaram que o efeito do tamanho da muda foi sempre mais evidenciado na primeira época de plantio.
Informações técnicas para o cultivo do mangarito são escassas. Por isso, ao realizar introduções de novas espécies em regiões diferentes daquelas de seu habitat, mediante pesquisa ou exploração agrícola, deve-se lembrar que há necessidade de se considerar as diferentes e inúmeras interações entre os organismos nativos e seus ambientes, numa visão plena da complexidade e do âmbito de variabilidade, para manter em equilíbrio as relações de interferência. Aliado a isso deve ser considerado o ciclo das culturas, as características dos solos quanto à estrutura, textura e fertilidade, populações de plantas e outros fatores.

A população de plantas tem efeito marcante sobre a produção, já que a intercompetição por água, luz e nutrientes, em plantios densos, pode contribuir para a redução da capacidade produtiva das plantas, incidindo em maior ou menor grau na produtividade das diferentes espécies (HEREDIA ZÁRATE et al., 1995). Isso porque, a maximização da produção depende, dentre outros fatores, da população empregada, que é função da capacidade suporte do meio e do sistema de produção adotado; do índice e da duração da área foliar fotossinteticamente ativa; da prolificidade da cultivar (BÜLL, 1993); da época de semeadura visando satisfazer a cinética de desenvolvimento e crescimento; bem como da adequada distribuição espacial de plantas na área, em conformidade com as características genotípicas (HEREDIA ZÁRATE et al., 2002).

Heredia Zárate et al. (2005a), avaliando a capacidade produtiva do mangarito 'Comum', sob três e quatro fileiras por canteiro e três espaçamentos entre plantas $(10,15$ e 20 $\mathrm{cm})$, observaram que as maiores produções total $\left(7,02 \mathrm{t} \mathrm{ha}^{-1}\right)$, líquida $\left(6,74 \mathrm{t} \mathrm{ha}^{-1}\right)$ e de rizomas não-comerciais $\left(4,06 \mathrm{t} \mathrm{ha}^{-1}\right)$ foram do tratamento quatro fileiras e $15 \mathrm{~cm}$ entre plantas e as de rizomas comerciais foram dos tratamentos quatro fileiras e $10 \mathrm{~cm}$ entre plantas $\left(2,97 \mathrm{tha}^{-1}\right)$ e três fileiras e 20 $\mathrm{cm}$ entre plantas $\left(2,90 \mathrm{t} \mathrm{ha}^{-1}\right)$. Já, as menores produções foram do tratamento quatro fileiras e $20 \mathrm{~cm}$ entre plantas (4,48; 4,27; 1,61 e 2,66 t ha ${ }^{-1}$, respectivamente).

Em razão do exposto, este trabalho foi desenvolvido em Dourados-MS, com o objetivo de avaliar a capacidade produtiva do mangarito cv. Comum, proveniente de rizomassemente secundários de tamanhos diferentes e cultivados sob três ou quatro fileiras de plantas no canteiro.

\section{MATERIAL E MÉTODOS}

O trabalho foi desenvolvido na Universidade Federal de Mato Grosso do Sul - UFMS, em DouradosMS, entre 5/9/2003 e 1/7/2004, em Latossolo Vermelho

Ciênc. agrotec., Lavras, v. 30, n. 5, p. 907-913, set./out., 2006 
distroférrico, de textura argilosa, com as seguintes características químicas: $4,9 \mathrm{de} \mathrm{pH}$ em $\mathrm{CaCl}_{2} ; 34,0 \mathrm{~g} \mathrm{dm}^{-3} \mathrm{de}$ M.O; 100,0 e $34,0 \mathrm{mg} \mathrm{dm}^{-3}$ de P e S, respectivamente, e 2,4; 36,0 e $19,0 \mathrm{mmol}_{\mathrm{c}} \mathrm{dm}^{-3}$ de $\mathrm{K}, \mathrm{Ca}$ e $\mathrm{Mg}$, respectivamente. O município de Dourados situa-se em latitude de $22^{\circ} 13^{\prime} 16^{\prime \prime S}$, longitude de $54^{\circ} 17^{\prime} 01^{\prime \prime} \mathrm{W}$ e altitude de $430 \mathrm{~m}$. O clima da região, segundo a classificação de Köppen é Mesotérmico Úmido, do tipo Cwa, com temperaturas e precipitações médias anuais variando de $20^{\circ}$ a $24^{\circ} \mathrm{C}$ e de $1250 \mathrm{~mm}$ a 1500 $\mathrm{mm}$, respectivamente.

Os tratamentos foram constituídos pela combinação dos arranjos das plantas em três e quatro fileiras por canteiro e do plantio de quatro tamanhos de rizomassemente secundários (Tabela 1), arranjados como fatorial $2 \times 4$, no delineamento experimental de blocos casualizados, com quatro repetições. Cada parcela teve área total de 2,7 $\mathrm{m}^{2}$ (1,5 $\mathrm{m}$ de largura e $1,8 \mathrm{~m}$ de comprimento) e área útil de $1,8 \mathrm{~m}^{2}$ (canteiro de $1,0 \mathrm{~m}$ de largura e 1,8 $\mathrm{m}$ de comprimento).

O solo da área do experimento foi preparado com aração, gradagem e levantamento de canteiros com rotoencanteirador. Para o plantio, foram abertos sulcos de aproximadamente $0,05 \mathrm{~m}$ de largura $\mathrm{x} 0,05 \mathrm{~m}$ de profundidade, a $33,3 \mathrm{~cm}$ e $25,0 \mathrm{~cm}$ para três e quatro fileiras de plantio, respectivamente. As mudas foram colocadas com espaçamentos de $15,0 \mathrm{~cm}$, no fundo dos sulcos, com os ápices para cima e posteriormente cobertas com a terra extraída.

As irrigações foram feitas por aspersão, com turnos de rega de três a quatro dias, de forma a manter o solo com umidade em torno de $70 \%$ da capacidade de campo. As capinas foram feitas com auxílio de enxada, quando necessárias. Não se observou a incidência de pragas nem de doenças, por isso, não foi necessária a utilização de agrotóxicos. A colheita foi realizada aos 300 dias após o plantio, quando as plantas das diferentes parcelas tinham, no mínimo, $90 \%$ da parte aérea com sintomas de senescência. Na colheita, foram avaliadas as produções de massas frescas total, de rizomas-mãe e de rizomas-filho, incluindo as produções das classes grandes $(>5,1 \mathrm{~g})$, médias (entre 2,1 e $4,9 \mathrm{~g}$ ) e pequenas $(<2,00 \mathrm{~g})$. Também foram determinadas as produções líquida (produção total menos gasto de rizomas-semente) e comercial (rizomasmãe mais rizomas-filho grandes) e não-comercial (rizomasfilho médios e pequenos menos o gasto de rizomassemente). Além disso, foram determinados os diâmetros, comprimentos e massas frescas médias dos rizomas. Os dados obtidos foram submetidos à análise de variância e as médias de tratamento comparadas pelo teste Tukey, a $5 \%$ de probabilidade.

\section{RESULTADOS E DISCUSSÃO}

As produções de massa fresca dos rizomas total, rizomas-mãe e rizomas-filho, foram influenciadas significativamente pela interação entre número de fileiras de plantas no canteiro e tamanho dos rizomas-semente utilizados no plantio (Tabela 2). As maiores produções das plantas cultivadas sob três fileiras foram daquelas originadas dos rizomas com massa de aproximadamente $3,0 \mathrm{~g}$. Nas plantas cultivadas sob quatro fileiras, as maiores produções foram das originadas dos rizomas com massa média de 1,3 a 3,0 g. Já, as menores produções foram das plantas originadas dos rizomas-semente de tamanho 4 , nos dois arranjos de plantas. Esses resultados assemelham-se aos obtidos por Vasconcelos (1972), citado por Monteiro \& Peressin (1997), que, estudando a influência do tamanho da muda sobre a produção de rizomas secundários do tipo comercial, obteve melhores resultados quando utilizou mudas do tipo rizoma secundário de cerca de $4,5 \mathrm{~g}$, em comparação com as de aproximadamente $1,5 \mathrm{~g}$.

As médias dos diâmetros, comprimentos e massas frescas dos rizomas-mãe $(43,13 \mathrm{~mm} ; 21,36 \mathrm{~mm}$ e $13,21 \mathrm{~g}) \mathrm{e}$ dos rizomas-filho, nas classes grande $(37,15 \mathrm{~mm} ; 18,57 \mathrm{~mm}$ e 9,60 g), média ( $30,71 \mathrm{~mm} ; 14,18 \mathrm{~mm}$ e 4,14 g) e pequena $(19,72 \mathrm{~mm} ; 9,46 \mathrm{~mm}$ e $1,07 \mathrm{~g})$, apresentaram variações significativas dependentes da interação entre número de fileiras no canteiro e tamanho de rizomas-semente, dentro de cada característica avaliada nos rizomas.

Os diâmetros, comprimentos e massas frescas médias dos rizomas-mãe e dos rizomas-filho, nas classes grandes, médias e pequenas, apresentaram valores característicos para cada tipo de rizoma (Tabela 3). Esses resultados sugerem que os rizomas-mãe já tinham alcançado a maturidade e o máximo crescimento, com provável aumento da translocação dos fotoassimilados da parte aérea para os rizomas-mãe e destes diretamente para os rizomas-filho ou que houve equilíbrio de translocação no tempo, entre a parte aérea e os rizomas. Hipótese semelhante foi levantada por Heredia Zárate (1988) para o taro (Colocasia esculenta (L.) Schott).

As variações quantitativas observadas dentro de cada característica avaliada nos rizomas das diferentes classes, foram dependentes significativamente da interação entre número de fileiras no canteiro e tamanho de rizomassemente (Tabela 3). Os maiores valores foram obtidos, na sua maioria, nos rizomas das plantas originadas dos rizomas-semente de tamanho 1 e cultivadas sob três fileiras, exceto para comprimento e massa fresca de rizoma-mãe, diâmetro de rizoma-filho médio e diâmetro e massa fresca 
de rizoma-filho pequeno. Já, os menores valores foram das plantas originadas dos rizomas-semente de tamanho 4, nos dois arranjos de plantas. Esses resultados sugerem que os sistemas vegetais têm mecanismos de autoregulação, baseados na capacidade de adaptação do organismo individual e das populações ou no equilíbrio das relações de interferência, como competição por nutrientes, água e outros (LARCHER, 2000; TAIZ \& ZEIGER, 2004).

No final do ciclo vegetativo, houve, em média, 96,88\% (149.040 plantas ha-1); 73,64\% (113.290 plantas $\left.\mathrm{ha}^{-1}\right) ; 57,17 \%$ (87.950 plantas ha-1) e 42,91\% (66.010 plantas $\mathrm{ha}^{-1}$ ) de plantas provenientes dos tamanhos 2, 1, 3 e 4, respectivamente (153.845 plantas ha-1). Em relação ao número de linhas de plantas no canteiro, a sobrevivência foi de $66,07 \%$ (87.130 plantas ha $\left.{ }^{-1}\right)$ e $68,82 \%$ (121.010 plantas ha-1) para três (131.868 plantas ha-1) e quatro linhas (175.824 plantas $\mathrm{ha}^{-1}$ ), respectivamente. As maiores produções total $\left(11,48 \mathrm{t} \mathrm{ha}^{-1}\right)$, líquida $\left(11,09 \mathrm{t} \mathrm{ha}^{-1}\right) \mathrm{e}$ de rizomas comerciais $\left(4,51 \mathrm{t} \mathrm{ha}^{-1}\right)$ foram do tratamento três fileiras de plantas e plantio de rizomas de tamanho 1 (Tabela 4) e as de rizomas não-comerciais foram do tratamento quatro fileiras de plantas e plantio de rizomas de tamanho $2\left(7,41 \mathrm{t} \mathrm{ha}^{-1}\right)$. As menores produções total $\left(0,96 \mathrm{t} \mathrm{ha}^{-1}\right)$, líquida $\left(0,86 \mathrm{tha}^{-1}\right)$ e de rizomas comerciais $\left(0,60 \mathrm{t} \mathrm{ha}^{-1}\right)$ e não-comerciais $\left(0,26 \mathrm{t} \mathrm{ha}^{-1}\right)$ foram do tratamento três fileiras de plantas e plantio de rizomas de tamanho 4. Com base nesses resultados, supõe-se que a quantidade de reservas presentes nas mudas influenciou as capacidades de brotação dos rizomas e de sobrevivência das plântulas, além da produção de rizomas das plantas provenientes de cada tipo de muda (Tabela 3), por serem elas consideradas importante fator relacionado ao crescimento e produção do mangarito (HEREDIA ZÁRATE et al., 2005b).

TABELA 1 - Médias de massa fresca e de diâmetro dos rizomas-semente, populações e gasto de rizomas do mangarito cv. Comum, cultivado sob três e quatro fileiras de plantas no canteiro e quatro tamanhos de rizomas-semente. Dourados, UFMS, 2003 - 2004.

\begin{tabular}{|c|c|c|c|c|c|}
\hline \multicolumn{2}{|c|}{ Fatores } & \multicolumn{2}{|c|}{ Rizoma-semente* } & \multirow{2}{*}{$\begin{array}{c}\text { População } \\
\left(\text { plantas } \text { ha }^{-1}\right)\end{array}$} & \multirow{2}{*}{$\begin{array}{c}\text { Gasto de } \\
\text { rizomas } \\
\left(\mathrm{kg} \mathrm{ha}^{-1}\right)^{*}\end{array}$} \\
\hline $\begin{array}{c}\text { Fileiras no } \\
\text { canteiro }\end{array}$ & $\begin{array}{c}\text { Tamanho rizoma- } \\
\text { semente }\end{array}$ & Massa fresca (g) & Diâmetro (mm) & & \\
\hline \multirow{4}{*}{3} & 1 & 2,96 & 8,77 & \multirow{4}{*}{131.868} & 390,33 \\
\hline & 2 & 1,33 & 6,05 & & 175,38 \\
\hline & 3 & 1,13 & 5,68 & & 149,01 \\
\hline & 4 & 0,78 & 4,83 & & 102,86 \\
\hline \multirow{4}{*}{4} & 1 & 2,96 & 8,77 & \multirow{4}{*}{175.824} & 520,44 \\
\hline & 2 & 1,33 & 6,05 & & 233,85 \\
\hline & 30 & 1,13 & 5,68 & & 198,68 \\
\hline & 4 & 0,78 & 4,83 & & 137,14 \\
\hline
\end{tabular}

* Média de 300 rizomas em cada tamanho.

** População x massa fresca do rizoma semente. 


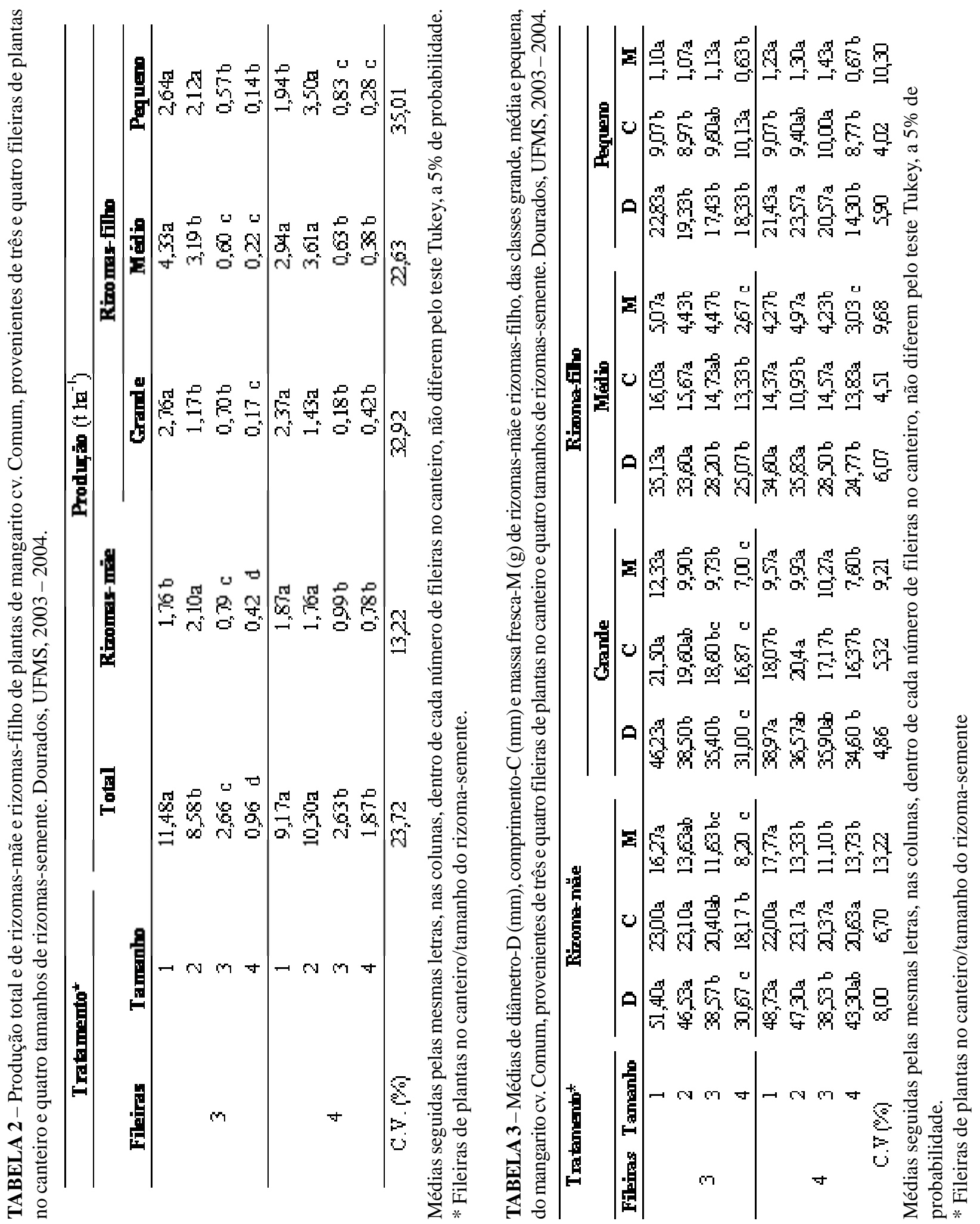

Ciênc. agrotec., Lavras, v. 30, n. 5, p. 907-913, set./out., 2006 
TABELA 4 - Médias de rizomas-semente gastos e de produções de rizomas total, líquida, comercial e não-comercial das plantas do mangarito cv. Comum, em função de três e quatro fileiras de plantas no canteiro e quatro tamanhos de rizomas-semente. Dourados, UFMS, 2003 - 2004.

\begin{tabular}{|c|c|c|c|c|c|c|}
\hline \multicolumn{2}{|c|}{ Tratamento* } & \multirow{2}{*}{$\begin{array}{c}\text { Rizoma } \\
\text { semente } \\
\left(\mathrm{kg} \mathrm{ha}^{-1}\right)^{* *}\end{array}$} & \multicolumn{4}{|c|}{ Produção (t ha-1) } \\
\hline Fileiras & Tamanho & & Total & Líquida & Comercial & Não-comercial \\
\hline \multirow{4}{*}{3} & 1 & 390,33 & $11,48 \mathrm{a}$ & 11,09 & $4,51 \mathrm{a}$ & 6,58 \\
\hline & 2 & 175,38 & $8,58 \mathrm{~b}$ & 8,40 & $3,27 \mathrm{a}$ & 5,13 \\
\hline & 3 & 149,01 & $2,66 \mathrm{c}$ & 2,51 & $1,49 \mathrm{~b}$ & 1,02 \\
\hline & 4 & 102,86 & $0,96 \mathrm{~d}$ & 0,86 & $0,60 \mathrm{~b}$ & 0,26 \\
\hline \multirow{4}{*}{4} & 1 & 520,44 & $9,17 \mathrm{a}$ & 8,65 & $4,24 a$ & 4,41 \\
\hline & 2 & 233,85 & $10,30 \mathrm{a}$ & 10,06 & $3,19 \mathrm{a}$ & 6,88 \\
\hline & 3 & 198,68 & $2,63 \mathrm{~b}$ & 2,43 & $1,17 \mathrm{~b}$ & 1,26 \\
\hline & 4 & 137,14 & $1,87 \mathrm{~b}$ & 1,73 & $1,20 \mathrm{~b}$ & 0,53 \\
\hline \multicolumn{2}{|c|}{ C.V. (\%) } & - & 23,72 & - & 33,24 & - \\
\hline
\end{tabular}

Médias seguidas pelas mesmas letras, nas colunas, dentro de cada número de fileiras no canteiro, não diferem pelo teste de Tukey, a $5 \%$ de probabilidade.

* Fileiras de plantas no canteiro/tamanho do rizoma-semente

** População do tratamento x massa média dos rizomas-semente (média de 1200 rizomas = 1,55 g)

Produção total $=$ Produção de rizomas-mãe + produção total de rizomas-filho

Produção líquida = Produção total de rizomas menos gasto de rizomas-semente

Produção comercial $=$ Produção de rizomas-mãe mais produção de rizomas-filho grande

Produção não comercial $=($ Produção de rizomas-filho médio + produção de rizomas-filho pequeno $)-($ gasto de rizomas-semente).

\section{CONCLUSÃO}

Nas condições em que foi conduzido o experimento e ao relacionar as produções total e comercial conclui-se que, foi melhor cultivar o mangarito 'Comum' sob três fileiras de plantas no canteiro e plantio de rizomas com massa fresca de $3,0 \mathrm{~g}$.

\section{AGRADECIMENTOS}

Ao $\mathrm{CNPq}$, pelas bolsas concedidas, e à FUNDECTMS, pelos recursos financeiros.

\section{REFERÊNCIAS BIBLIOGRÁFICAS}

APEZZATO-DA-GLÓRIA, B. Anatomia de sistemas subterrâneos. In: CONGRESSO LATINOAMERICANO DE RAÍZES TROPICAIS, 1.; CONGRESSO BRASILEIRO DE MANDIOCA, 9., 1996, São Pedro. Programa e resumos... São Pedro: CERAT/UNESP, 1996. p. 45-51.
BÜLL, L. T. Cultura do milho: fatores que afetam a produtividade. Piracicaba: POTAFOS, 1993. 301 p.

CEREDA, M. P. Agricultura: tuberosas amiláceas Latino Americanas. São Paulo: Fundação Cargill, 2002. $278 \mathrm{p}$.

HEREDIA ZÁRATE, N. A. Curvas de crescimento de inhame (Colocasia esculenta (L.) Schott), considerando cinco populações, em solo seco e alagado. 1988. 95 f. Tese (Doutorado em Fitotecnia) - Universidade Federal de Viçosa, Viçosa, 1988.

HEREDIA ZÁRATE, N. A.; ALVES SOBRINHO, T.; VIEIRA, M. C.; SUZUKI, M. T. Influência do espaçamento na cultura e na colheita semi-mecanizada de inhame. Horticultura Brasileira, Brasília, v. 13, n. 1, p. 59-60, 1995. 
HEREDIA ZÁRATE, N. A.; VIEIRA, M. C.; GRACIANO, J. D.; FIGUEIREDO, P. G. Produção do mangarito 'Comum' proveniente de oito tamanhos de rizomas-semente. In: CONGRESSO BRASILEIRO DE OLERICULTURA, 45.; CONGRESSO BRASILEIRO DE FLORICULTURA E PLANTAS ORNAMENTAIS, 15.; CONGRESSO BRASILEIRO DE CULTURA DE TECIDOS DE PLANTAS, 2., 2005, Fortaleza. Horticultura Brasileira, Brasília, v. 23, n. 2, 2005a. Suplemento. CD-ROM.

HEREDIA ZÁRATE, N. A.; VIEIRA, M. C.; MINUZZI, A. Brotação de seis tipos de mudas dos clones de inhame roxo e mimoso. Ciência e Agrotecnologia, Lavras, v. 26, n. 4, p. 699-704, 2002.

HEREDIA ZÁRATE, N. A.; VIEIRA, M. C.; PONTIM, B. C. A. Arranjo de plantas na produção do mangarito
(Xanthosoma mafaffa Schott) 'Comum'. Acta Scientiarum: Agronomy, Maringá, v. 27, n. 3, p. 409-413, 2005 b.

LARCHER, W. W. Ecofisiologia vegetal. São Carlos: RiMa Artes e Textos, 2000. 531 p.

MONTEIRO, D. A.; PERESSIN, V. A. Efeito do tamanho do rizoma-semente, da época e do local de plantio, na produção de rizomas de mangará. Bragantia, Campinas, v. 56, n. 1, p. 155-161, 1997.

SEDIYAMA, M. A. N.; CASALI, V. W. D. Propagação vegetativa da mandioquinha-salsa. Informe Agropecuário, Belo Horizonte, v. 19, n. 190, p. 24-27, 1997.

TAIZ, L.; ZEIGER, E. Fisiologia vegetal. 3. ed. Porto Alegre: Artmed, 2004. 720 p. 